\title{
A spectroscopy-based Age-Metallicity Relation of the SMC
}

\author{
Andrea Kayser ${ }^{1}$, Eva K. Grebel ${ }^{1}$, Daniel R. Harbeck ${ }^{2}$, \\ Andrew A. Cole ${ }^{3}$, Andreas Koch ${ }^{1,4}$, Katharina Glatt ${ }^{1}$, \\ John S. Gallagher ${ }^{2}$ and Gary S. Da Costa ${ }^{5}$ \\ ${ }^{1}$ Astronomical Institut, U. Basel, Venusstr. 7, 4102 Binningen, Switzerland \\ ${ }^{2}$ Dept. Astronomy, U. Wisconsin, 475 N. Carter St. Madison, WI 53706, USA \\ ${ }^{3}$ Astronomy Dept., U. Minnesota, 116 Church St. SE, MN 55455, USA \\ ${ }^{4}$ Dept. of Astronomy, U. of California, 430 Portola Plaza, Los Angeles, CA 90095-1547, USA \\ ${ }^{5}$ ANU, Mt Stromlo Observatory, Cotter Rd, Weston ACT 2611, Australia.
}

\section{Introduction}

The Small Magellanic Cloud (SMC) is the only dwarf galaxy in the Local Group that is known to have formed and preserved populous star clusters continuously over the past 12 Gyr. Due to its proximity $(\approx 60 \mathrm{kpc})$, stars can be resolved well below the oldest main sequence turnoff points. This facilitates accurate age and metallicity determinations without suffering from the age-metallicity degeneracy. Therefore, the SMC star clusters provide a unique closely spaced set of single-age, single-metallicity tracers to derive a well-sampled age-metallicity relation required for the understanding of the star formation history of this satellite galaxy. Up to date spectroscopically based metallicity estimates exist only for the small number of 7 clusters (Da Costa \& Hatzidimitriou 1998). Our project now more than doubles the available data set by the observation of 10 additional clusters.

\section{Data and Analysis}

We observed spectra of a large number of individual red giant stars in 12 star clusters in the SMC in the near infrared region, covering the prominent $\mathrm{Ca}$ II triplet (CaT) absorption features. The clusters observed are K 28, K 44, L 11, L 116, L 32, L 38, NGC 152, NGC 339, NGC 361, NGC 411, NGC 416 and NGC 419. For comparison two clusters were chosen to coincide with the Da Costa \& Hatzidimitriou (1998) sample (L 11 and NGC 339). The observations were carried out between October 2005 and July 2006 with the multi-object facility FORS2/MXU at the VLT at ESO/Paranal.

Candidate cluster members were selected from their radial velocities and position in the colour-magnitude diagram. Only those stars with measured velocities within the range of $10 \mathrm{~km} / \mathrm{s}(\approx 2 \sigma)$ from the clusters' mean heliocentric velocity (Kayser et al. in prep.) were chosen (Fig. 1a). As the Ca II triplet calibration is only well calibrated for red giants, we selected only stars on the upper red giant branch from each cluster (Fig. 1b). Furthermore we rejected stars with distances larger than 6 times the core radius from the clusters' centres.

Abundances were derived from $\Sigma \mathrm{W}$, the summed equivalent width of the infrared $\mathrm{Ca}$ II triplet absorption lines in each star. Apart from metallicity, this index is strongly dependent on stellar parameters, in particular log g. In order to extract the metallicity information $\Sigma \mathrm{W}$ is plotted vs. the magnitude difference from the horizontal branch $\left(V-V_{H B}\right)$. In this diagram the calibration by Rutledge et al. (1997) and Cole et al. 

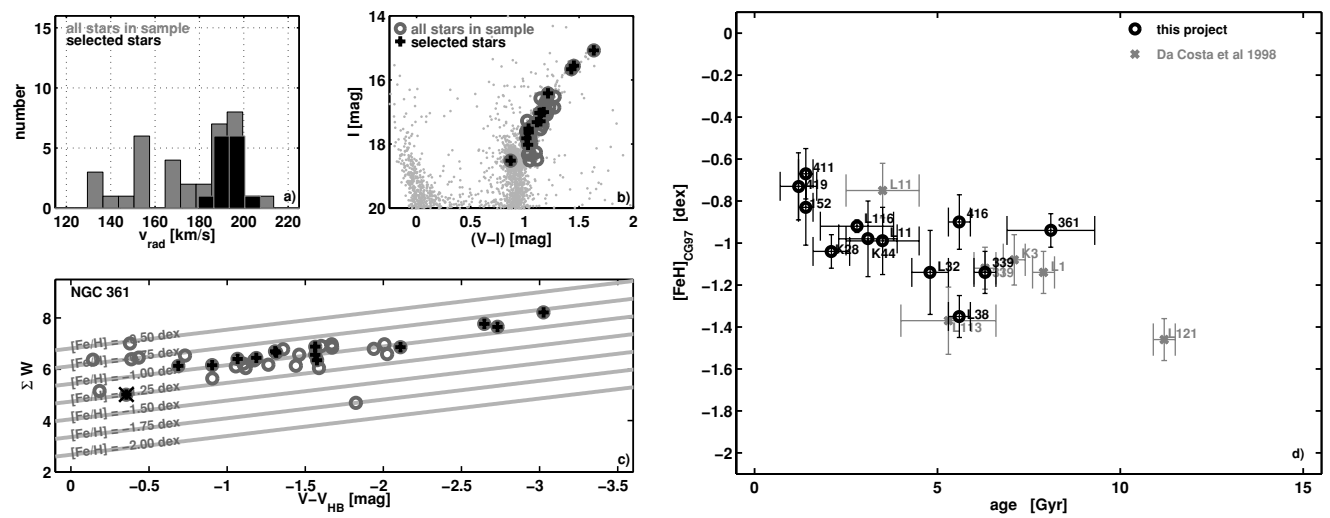

Figure 1. In a) and b) we show the membership selection for the example of NGC 361. c) indicates the position of the stars in the calibrating plane. The age-metallicity relation of the $\mathrm{SMC}$ based on CaT measurements is given in d).

(2004) provided a set of isometallicity lines, which we find to be in good agreement with our calibration clusters. We then derived metallicities adopting the scale of Carretta \& Gratton (1997).

\section{Results}

Using the CaT technique we can derive $[\mathrm{Fe} / \mathrm{H}]$ values for all 12 clusters in our sample with a mean accuracy of 0.13 dex. For the 2 clusters in common with the sample of Da Costa \& Hatzidimitriou (1998) (L 11 and NGC 339) we find good agreement within the errors.

Combing these newly derived metallicities with age estimates found in the literature (e.g.: de Freitas Pacheco et al. 1998, Mighell et al. 1998, Piatti et al. 2001) and our own HST/ACS data (Glatt et al. in prep.) we can for the first time present a well-sampled agemetallicity relation for the SMC that is fully based on spectroscopic metallicity estimates (Fig. 1d). This relation shows that at a given age there may be a metallicity spread of up to 0.4 dex, which exceeds the uncertainties in $[\mathrm{Fe} / \mathrm{H}]$ by a factor of 3 . Evidently in the past the SMC was not well mixed. This is rather unusual for a dwarf galaxy like this which is expected to be thoroughly mixed by expanding gas-shells driven by evolving massive stars (Da Costa \& Hatzidimitriou 1998). Interestingly the largest scatter in $[\mathrm{Fe} / \mathrm{H}]$ falls in the regime of the prominent age gap in the LMC cluster distribution. We conclude that the SMC has obviously experienced a complex star formation history.

\section{References}

Armandroff, T. E. \& Da Costa, G. S., 1991:AJ 101, 1329

Carretta, E. \& Gratton, R. G. 1997: A\&AS 121, 95

Cole et al. 2004: MNRAS 347, 367

Da Costa, G. S. \& Hatzidimitriou, D. 1998: AJ 115, 1934

de Freitas Pacheco, J. A., Barbuy, B. \& Idiart, T. 1998: A $\&$ A 332, 19

Mighell, K. J., Sarajedini, A. \& French, R. S. 1998: AJ 116, 2395

Piatti, A. E. et al. 2001: MNRAS 325, 792

Rutledge, G. A., Hesser, J. E. \& Stetson, P. B. 1997: PASP 109, 907 